\title{
Balanced Truncation for a General Class of Discrete-Time Markov Jump Linear Systems
}

\author{
Georgios Kotsalis, Anders Rantzer
}

\begin{abstract}
This paper develops a generalization of the balanced truncation algorithm applicable to discrete-time Markov jump linear systems. The approximation error, which is captured by means of the stochastic $L_{2}$ gain, is bounded from above by twice the sum of singular numbers associated to the truncated states of each mode.
\end{abstract}

\section{INTRODUCTION}

\section{A. Previous Work on Model Reduction}

The concept of model reduction is pervasive in all areas where system theoretic ideas have been applied. The objective is to find an adequate simplified model for a given complex system. A particular reduction algorithm is judged upon the certificates it provides concerning the level of complexity reduction achieved, the accuracy of the reduced order model and the algorithmic cost involved.

In the context of linear time-invariant (abbreviated LTI) systems a distance measure relevant to robustness analysis is the $H_{\infty}$ system norm of the error system between the original and reduced order model. Balanced truncation and optimal Hankel model reduction are two related reduction algorithms, which are suboptimal, but are accompanied by provable a priori bounds to the aforementioned metric.

Balanced realizations were originally proposed in the controls literature in [1]. Optimal Hankel model reduction was developed in [2] and is inspired by the results in [3]. The derivation of the associated error bounds in continuous and discrete-time settings can be found in [2], [4], [5] and [6]. Both methods are based on the computation of the singular values of the Hankel operator of an LTI system and are frequently termed as SVD based approximation methods [7]. Their strength lies in the guarantees of quality they provide and their main drawback is the fact that they require computations of the order $O\left(n^{3}\right)$ and storage of the order $O\left(n^{2}\right)$, where $n$ is the number of states of the original system. A possibility of overcoming this deficit to some extent,

G. Kotsalis is with Department of Electrical and Computer Engineering, Georgia Institute of Technology, Atlanta, GA, USA georgios.kotsalis@ece.gatech.edu

A. Rantzer is with Department of Automatic Control, Lund University, Lund, Sweden rantzerdcontrol.1th.se is to seek approximate balanced representations, see [8] and [9] for some early references on the subject.

Balanced truncation has been investigated also outside the realm of LTI systems. In [10] a generalization to multidimensional and uncertain systems in the linearfractional framework is presented. Linear parametervarying systems are investigated in [11]. The case of linear time-varying systems is the subject of [12] where an operator-theoretic framework is employed to give bounds similar to those that apply to time-invariant models. Later more general error bounds for linear timevarying systems were obtained in [13].

\section{B. Previous Work on Markov Jump Linear Systems}

Jump linear systems (abbreviated JLS's) are abstractions of hybrid systems, which combine continuous and discrete dynamics. They form an extension of LTI systems, in the sense that the coefficients of the linear transformation are functions of parameters. The transition between the different modes of operation is controlled by an exogenous parametric input, which is frequently referred to as the switching signal. In this work it is assumed that the switching signal takes values in a finite set and that it follows an unconstrained stochastic evolution. There is a large body of literature in the fields of econometrics and system theory pertaining to the class of JLS's with randomly varying parameters. Fundamental contributions on the jump linear control problem in a continuous-time setting can be found in [14], [15] and [16]. Subsequently various analysis and synthesis results applicable to linear time-invariant systems have been extended to to the class of Markov jump linear systems (abbreviated MJLS's). A comprehensive review of this material can be found in [17] and the references therein. Stochastic JLS's arise naturally in connection with networked control systems when the effects of variable sampling rates, link failures, delays and other communication constraints are modeled in a probabilistic framework. A review article on networked control systems is [18]. Model reduction of MJLS's is the topic of [19], where the search of an optimal, in terms of the stochastic $L_{2}$ gain, reduced ordered model is posed as a non convex optimization problem. 


\section{Contributions of the Paper}

The contribution of this paper consists of a balanced truncation algorithm for discrete-time Markov jump linear systems. The main point of the reduction algorithm is the formulation of two sets of dissipation inequalities, which in conjunction with a suitably defined storage function enable the derivation of suboptimal reduced order models, which are accompanied by a provable a priori upper bound on the stochastic $L_{2}$ gain of the approximation error. result can be considered as generalization of the corresponding balanced truncation algorithm for linear time-invariant systems.

\section{Notation}

The set of nonnegative integers is denoted by $\mathbf{N}$, the set of positive integers by $\mathbf{Z}_{+}$and the set of real numbers by $\mathbf{R}$. Let $n \in \mathbf{Z}_{+}$then $\mathbf{R}^{n}$ denotes the Euclidean $n$ space. The transpose of a column vector $x \in \mathbf{R}^{n}$ is $x^{\prime}$. Let $x \in \mathbf{R}^{n}$, then $|x|^{2}=x^{\prime} x$ denotes the square of the Euclidean norm. Let $P \in \mathbf{R}^{n \times n}$, then $P>0$ indicates that it is a positive definite matrix and the notation $|x|_{P}^{2}$ stands for $x^{\prime} P x$, the square of the weighted norm of $x \in \mathbf{R}^{n}$. The positive definite square root of $P$ is denoted by $P^{\frac{1}{2}}$. The identity matrix in $\mathbf{R}^{n \times n}$ is written as $I_{n}$. Let $A: \mathbf{R}^{n \times n}$, then $r_{\sigma}[A]$ denotes the spectral radius of $A$. Let $P, Q \in \mathbf{R}^{n \times n}$, the inner product of these two matrices is defined as $\langle P, Q\rangle=\operatorname{Tr}\left[P^{\prime} Q\right]$. Let $f$ : $\mathbf{N} \rightarrow \mathbf{R}^{n}$, the notation $f$ and $\{f(k)\}_{k=0}^{\infty}$ will be used interchangeably. The space of square summable vector sequences with elements in $\mathbf{R}^{n}$ is denoted by $l_{2}^{n}$. Let $f \in l_{2}^{n}$ then $\|f\|_{2}^{2}$ stands for $\sum_{k=0}^{\infty}|f(k)|^{2}$. The unit sphere in $l_{2}^{n}$ is denoted by $S_{2}^{n}=\left\{f \in l_{2}^{n}:\|f\|_{2}=1\right\}$. Let $x$ be a random variable, then its expected value is denoted by $\mathbf{E}[x]$.

\section{SySTEM MODEL}

The class of discrete-time Markov jump linear systems, abbreviated MJLS's, considered in this work is more general than what is standard in the literature, in the sense that the system matrices in the state space recursion exhibit stochastic dependence on the mode transition of the system. Apart from that, the dimension of the continuous valued part of the state variable is allowed to vary depending on which discrete mode the system resides in. This class of systems arises naturally in the area of networked control systems, when various constraints such as variable intersampling times, package losses and delays are modeled in a probabilistic framework. A directed graph with multiple edges between nodes is considered. In particular let $Q, N \in \mathbf{Z}_{+}$and define $\boldsymbol{\Theta}=\{1, \ldots, N\}$ as the set of nodes and $\mathbf{Q}=$ $\{1, \ldots, Q\}$ as the set of edges. Consider also the sets $\boldsymbol{\Phi}_{i j}, i, j \in \boldsymbol{\Theta}$, which contains the edges emanating from node $i$ and ending in node $j$ and let $n, m, r$ be maps from $\Theta$ into $\mathbf{Z}_{+}$. The state space representation of a MJLS $\mathbf{L}$ is

$$
\begin{aligned}
x(k+1) & =A[\phi(k+1)] x(k)+B[\phi(k+1)] f(k), \\
y(k) & =C[\theta(k)] x(k), k \in \mathbf{N} .
\end{aligned}
$$

The system mode, which can also be interpreted as an exogenous parametric input, is $\theta(k) \in \boldsymbol{\Theta}$ and corresponds to the state of a Markov chain, taking values in $\Theta$. The transition probability matrix of the Markov chain is denoted by $P=\left[p_{i j}\right], i, j \in \boldsymbol{\Theta}$, where $p_{i j}=$ $\mathbf{P}[\theta(k+1)=j \mid \theta(k)=i]$. The signal $\phi(k)$ is a sequence of random variables taking values in the finite set $\mathbf{Q}$. The distribution of $\phi(k+1)$ is determined by $\theta(k), \theta(k+1)$. Define the set $\mathcal{Y}_{k}=\{\theta(0), \ldots, \theta(k), \phi(1), \ldots, \phi(k)\}$ and let $F$ be a subset of $\mathbf{Q}$, then

$$
\begin{aligned}
& \operatorname{Pr}\left[\phi(k+1) \in F \mid \mathcal{Y}_{k}, \theta(k+1)\right]= \\
& \operatorname{Pr}[\phi(k+1) \in F \mid \theta(k), \theta(k+1)] .
\end{aligned}
$$

The relevant conditional probabilities are denoted by $q_{l}$, where $l \in \mathbf{Q}, i, j \in \boldsymbol{\Theta}$ and $q_{l}=\operatorname{Pr}[\phi(k+1)=$ $l \mid \theta(k)=i, \theta(k+1)=j]$. The continuous valued part of the state variable is $x(k) \in \mathbf{R}^{n[\theta(k)]}$, note that its dimension depends on $\theta(k)$. Similarly the number of sensors and actuators depends on the mode the system is in, thus the input signal $f(k) \in \mathbf{R}^{r[\theta(k)]}$ and output $y(k) \in \mathbf{R}^{m[\theta(k)]}$. Throughout the work the input signal is taken to be deterministic.

\section{StABILITY}

There are several stability notions for stochastic systems. The relevant concept to this work is that of mean square stability.

Definition 3.1: [20] The MJLS L with $\{f(k)\}=\{0\}$ is mean square stable, if for every initial condition $\theta(0) \in$ $\boldsymbol{\Theta}, x(0) \in \mathbf{R}^{n[\theta(0)]}$

$$
\mathbf{E}\left[|x(k)|^{2}\right] \rightarrow 0 \text { as } k \rightarrow \infty .
$$

Theorem 3.1: Let $F[i]>0, F[i] \in \mathbf{R}^{n[i] \times n[i]}, i \in \boldsymbol{\Theta}$. The MJLS $\mathbf{L}$ is mean square stable iff there exists a unique $N$-tuple of positive definite matrices $G[i]>$ $0, G[i] \in \mathbf{R}^{n[i] \times n[i]}, i \in \boldsymbol{\Theta}$ such that :

$$
G[i]-\sum_{j \in \boldsymbol{\Theta}} p_{i j} \sum_{l \in \boldsymbol{\Phi}_{i j}} q_{l} A[l]^{\prime} G[j] A[l]=F[i], \quad \forall i \in \mathbf{\Theta}
$$


Proof: "๘" The above relation implies that $V[x, \theta]=|x|_{G[\theta]}^{2}$ acts as a stochastic Lyapunov function for the system $\mathbf{L}$. Consider the unforced evolution of the system $\mathbf{L}$

$$
x(k+1)=A[\phi(k+1)] x(k),
$$

and let

$$
\hat{G}[i]=\sum_{j \in \boldsymbol{\Theta}} p_{i j} \sum_{l \in \boldsymbol{\Phi}_{i j}} q_{l} A[l]^{\prime} G[j] A[l],
$$

then one has

$\mathbf{E}[V[x(k+1), \theta(k+1)] \mid x(k), \theta(k)]=x(k)^{\prime} \hat{G}[\theta(k)] x(k)$.

Relation (2) implies then,

$\mathbf{E}[V[x(k+1), \theta(k+1)] \mid x(k), \theta(k)]-V[x(k), \theta(k)]<0$.

By making use of the law of iterated expectations, namely for any two random variables, $x, y, \mathbf{E}[\mathbf{E}[x \mid y]]=$ $\mathbf{E}[x]$, one gets from the above relation

$$
\mathbf{E}[V[x(k+1), \theta(k+1)]-V[x(k), \theta(k)]]<0 .
$$

Thus

$$
\mathbf{E}[V[x(k), \theta(k)]] \rightarrow 0 \text { as } k \rightarrow \infty
$$

and since $G[\theta(k)]>0, \forall \theta(k) \in \boldsymbol{\Theta}$ mean square stability follows.

$" \rightarrow "$ Define the mode indicator function

$$
I_{i}(k)= \begin{cases}1 & \text { if } \theta(k)=i \\ 0 & \text { otherwise }\end{cases}
$$

and note that

$$
\mathbf{E}\left[|x(k)|^{2}\right]=\mathbf{E}\left[\sum_{i \in \boldsymbol{\Theta}}|x(k)|^{2} I_{i}(k)\right] .
$$

Let $X_{i}(k)=\mathbf{E}\left[x(k) x(k)^{\prime} I_{i}(k)\right]$ then the mean square stability condition implies $X_{i}(k) \rightarrow 0$ as $k \rightarrow \infty$, where $X_{j}(k+1)=\sum_{i \in \boldsymbol{\Theta}} p_{i j} \sum_{l \in \boldsymbol{\Phi}_{i j}} q_{l} A[l] X_{i}(k) A[l]^{\prime}, \quad \forall j \in \boldsymbol{\Theta}$.

Define $\mathbf{H}^{n}=\mathbf{R}^{n[1] \times n[1]} \times \ldots \times \mathbf{R}^{n[N] \times n[N]}$ and introduce the linear operator $\mathcal{T}: \mathbf{H}^{n} \rightarrow \mathbf{H}^{n}$, where

$$
\mathcal{T}[V]=\left[\ldots, \sum_{i \in \boldsymbol{\Theta}} p_{i j} \sum_{l \in \boldsymbol{\Phi}_{i j}} q_{l} A[l] V[i] A[l]^{\prime}, \ldots\right] .
$$

Using this notation one can write compactly

$$
\begin{aligned}
& \mathcal{T}\left[\left[X_{1}(k), \ldots, X_{N}(k)\right]\right]= \\
& {\left[X_{1}(k+1), \ldots, X_{N}(k+1)\right]}
\end{aligned}
$$

and consequently mean square stability implies $r_{\sigma}[\mathcal{T}]<$ 1 , where $r_{\sigma}$ denotes the spectral radius. Similarly define the linear operator $\mathcal{L}: \mathbf{H}^{n} \rightarrow \mathbf{H}^{n}$, where

$$
\mathcal{L}[V]=\left[\cdots, \sum_{j \in \boldsymbol{\Theta}} p_{i j} \sum_{l \in \boldsymbol{\Phi}_{i j}} q_{l} A[l]^{\prime} V[j] A[l], \ldots\right] .
$$

The set of equations in (2) can be written as

$$
\begin{aligned}
& \mathcal{L}[[G[1], \ldots, G[N]]]-[G[1], \ldots, G[N]]= \\
& -[F[1], \ldots, F[N]]
\end{aligned}
$$

Let the inner product of $V, S \in \mathbf{H}^{n}$ be given by $<$ $V, S>=\sum_{i \in \Theta} \operatorname{Tr}\left[V_{i}^{\prime} S_{i}\right]$, then $\mathcal{L}^{\prime}=\mathcal{T}$.

$$
\begin{aligned}
& <\mathcal{T}[V], S>=\sum_{j \in \boldsymbol{\Theta}} \operatorname{Tr}\left[\mathcal{T}_{j}[V]^{\prime} S_{j}\right]=\sum_{j \in \boldsymbol{\Theta}} \operatorname{Tr}\left[\mathcal{T}_{j}\left[V^{\prime}\right] S_{j}\right] \\
= & \sum_{j \in \boldsymbol{\Theta}} \sum_{i \in \boldsymbol{\Theta}} p_{i j} \sum_{l \in \boldsymbol{\Phi}_{i j}} q_{l} \operatorname{Tr}\left[A[l] V[i]^{\prime} A[l]^{\prime} S_{j}\right] \\
= & \sum_{j \in \boldsymbol{\Theta}} \sum_{i \in \boldsymbol{\Theta}} p_{i j} \sum_{l \in \boldsymbol{\Phi}_{i j}} q_{l} \operatorname{Tr}\left[V[i]^{\prime} A[l]^{\prime} S_{j} A[l]\right] \\
= & \sum_{i \in \boldsymbol{\Theta}} \sum_{j \in \boldsymbol{\Theta}} p_{i j} \sum_{l \in \boldsymbol{\Phi}_{i j}} q_{l} \operatorname{Tr}\left[V[i]^{\prime} A[l]^{\prime} S_{j} A[l]\right] \\
= & \sum_{i \in \boldsymbol{\Theta}} \operatorname{Tr}\left[V[i]^{\prime} \mathcal{L}_{i}[S]\right] \\
= & <V, \mathcal{L}[S]>.
\end{aligned}
$$

Since $\mathcal{L}$ is the adjoint of $\mathcal{T}$ one has $r_{\sigma}[\mathcal{T}]<1 \rightarrow$ $r_{\sigma}[\mathcal{L}]<1$ and

$$
G=\sum_{i=1}^{\infty} \mathcal{L}^{i}[F]>0
$$

is the unique positive definite solution to $\mathcal{L}[G]-G=$ $-F$.

Definition 3.2: The stochastic $L_{2}$ gain for the MJLS $\mathbf{L}$ is denoted by $\gamma_{\mathbf{L}}$ and is defined by

$$
\gamma_{\mathbf{L}}^{2}=\sup _{\theta(0) \in \boldsymbol{\Theta}} \sup _{f \in S_{2}^{m}} \sum_{k=0}^{\infty} \mathbf{E}\left[|y(k)|^{2}\right]
$$

under the assumption $x(0)=0, \theta(0) \in \mathbf{\Theta}$.

Lemma 3.1: Given is a MJLS $\mathbf{L}$ and let $\gamma \in \mathbf{R}, \gamma>$ 0 . Consider the quadratic function $V[x, \theta]=x^{\prime} G[\theta] x$, where $G[\theta] \in \mathbf{R}^{n[\theta] \times n[\theta]}, G[\theta]>0$ and $x \in \mathbf{R}^{n[\theta]}$. Suppose that

$$
\begin{aligned}
& \gamma^{2}|f|^{2}+V[x, i] \geq \\
& |C[i] x|^{2}+\sum_{j \in \boldsymbol{\Theta}} p_{i j} \sum_{l \in \boldsymbol{\Phi}_{i j}} q_{l} V[A([l] x+B[l] f, j], \\
& \forall x \in \mathbf{R}^{n[i]}, \forall f \in \mathbf{R}^{m}, \forall i \in \mathbf{\Theta}
\end{aligned}
$$

then the stochastic $L_{2}$ gain of $\mathbf{L}$ does not exceed $\gamma$. 
Theorem 3.2: If the MJLS $\mathbf{L}$ is mean square stable, then its stochastic $L_{2}$ gain is finite.

Proof: Lemma 3.1 will be employed in this proof. Note that (3) can be written equivalently in matrix form as

$$
W[i] \leq\left[\begin{array}{cc}
G[i] & 0 \\
0 & \gamma^{2} I_{m}
\end{array}\right], \forall i \in \mathbf{\Theta}
$$

where

$$
\begin{aligned}
& W[i]=\left[\begin{array}{ll}
W_{11}[i] & W_{12}[i] \\
W_{12}^{\prime}[i] & W_{22}[i]
\end{array}\right]= \\
& \sum_{j \in \Theta} p_{i j} \sum_{l \in \boldsymbol{\Phi}_{i j}} q_{l}\left[\begin{array}{cc}
A[l] & B[l] \\
C[i] & 0
\end{array}\right]^{\prime}\left[\begin{array}{cc}
G[j] & 0 \\
0 & I_{p}
\end{array}\right] \\
& {\left[\begin{array}{cc}
A[l] & B[l] \\
C[i] & 0
\end{array}\right]}
\end{aligned}
$$

By taking the Schur complement one obtains the following set of sufficient conditions for (4) to hold

$$
\begin{aligned}
W_{11}[i] & <G[i], \\
W_{22}[i]-W_{12}^{\prime}[i]\left(W_{11}[i]-G[i]\right)^{-1} W_{12}[i] & <\gamma^{2} I_{m}, \\
\forall i \in \mathbf{\Theta} . &
\end{aligned}
$$

Mean square stability implies existence of a positive definite matrix $\tilde{P}[i]>0$, such that $\sum_{j \in \boldsymbol{\Theta}} p_{i j} \sum_{l \in \boldsymbol{\Phi}_{i j}} A[l]^{\prime} \tilde{P}[j] A[l]-\tilde{P}[i]<0$. Set $G[i]=\alpha \tilde{P}[i]$ with $\alpha \in \mathbf{R}, \alpha \geq 1$. It is straight to see that the first set of conditions in (5) can be satisfied by taking $\alpha$ large enough. Subsequently note that for a fixed value of $\alpha$ the second set of conditions in (5) can always be satisfied by taking $\gamma$ large enough. Finally let $V[x, \theta]=x^{\prime} G[\theta] x$ and note that the assumptions of Lemma 3.1 are fulfilled.

A standing assumption in this work is that a given MJLS $\mathbf{L}$ is mean square stable.

\section{A. Reduction by state truncation}

This concept of model reduction by means of state truncation is developed for MJLS's. One starts out with the state space representation of a MJLS $\mathbf{L}$ as in (1) and applies an invertible coordinate transformation $x(k)=$ $T[\theta(k)] \tilde{x}(k)$ that puts the "most important" states in first components of the transformed state vector $\tilde{x}(k)$. The new state vector $\tilde{x}(k)$ is then partitioned as $\tilde{x}(k)=$ $\left[\tilde{x}_{1}(k)^{\prime}, \tilde{x}_{2}(k)^{\prime}\right]^{\prime}$, where $\tilde{x}_{1}(k) \in \mathbf{R}^{\hat{n}[\theta(k)]}$ corresponds to the states that are to be retained, $\tilde{x}_{2}(k) \in \mathbf{R}^{r[\theta(k)]}$ to the states that are to be removed and $\hat{n}[\theta(k)]=$ $n[\theta(k)]-r[\theta(k)]$. The state space matrices are partitioned accordingly as

$$
\begin{aligned}
\tilde{A}[\phi(k+1)] & =\left[\begin{array}{cc}
\tilde{A}_{11} & \tilde{A}_{12} \\
\tilde{A}_{21} & \tilde{A}_{22}
\end{array}\right][\phi(k+1)], \\
\tilde{B}[\phi(k+1)] & =\left[\begin{array}{c}
\tilde{B}_{1} \\
\tilde{B}_{2}
\end{array}\right][\phi(k+1)], \\
\tilde{C}[\theta(k)] & =\left[\begin{array}{cc}
\tilde{C}_{1} & \tilde{C}_{2}
\end{array}\right][\theta(k)], \theta(k) \in \mathbf{\Theta},
\end{aligned}
$$

and the state space representation of $\hat{\mathbf{L}}$ of order $\hat{n}$ is given by

$$
\begin{aligned}
\hat{x}(k+1) & =\hat{A}[\phi(k+1)] \hat{x}(k)+\hat{B}[\phi(k+1)] f(k), \\
\hat{y}(k) & =\hat{C}[\theta(k)] \hat{x}(k), k \in \mathbf{N}
\end{aligned}
$$

where

$$
\begin{gathered}
\hat{A}[\phi(k+1)]=\tilde{A}_{11}[\phi(k+1)], \hat{B}[\phi(k+1)]=\tilde{B}_{1}[\phi(k+1)], \\
\hat{C}[\theta(k)]=\tilde{C}_{1}[\theta(k)], \theta(k) \in \mathbf{\Theta}, \phi(k+1) \in \mathbf{Q} .
\end{gathered}
$$

Note that the size of the partitioned blocks of the state space matrices is varying depending on the number of states that have been truncated at each mode. For instance in the case where $\hat{n}[\theta(k)]=n[\theta(k)]$ and $\hat{n}[\theta(k+1)]=n[\theta(k+1)]$ then one has

$$
\hat{A}[\phi(k+1)]=\tilde{A}_{11}[\phi(k+1)]=\tilde{A}[\phi(k+1)]
$$

and so forth. In order to shorten subsequent notation, it will be convenient to think of the state variable of the reduced system submerged in the original state space. Let $r[\theta(k)]=n[\theta(k)]-\hat{n}[\theta(k)]$ and $\hat{x}_{1}(k)=$ $\left[\hat{x}(k)^{\prime}, 0^{\prime}\right]^{\prime} \in \mathbf{R}^{n[\theta(k)]}$, then in a slight abuse of notation $\hat{\mathbf{L}}$ will also be used to denote the system with state space representation

$$
\begin{aligned}
\hat{x}_{1}(k+1)= & \left(I_{n[\theta(k+1)]}-E_{r[\theta(k+1)]}\right) \\
& \left(\tilde{A}[\phi(k)] \hat{x}_{1}(k)+\tilde{B}[\phi(k+1)] f(k)\right) \\
\hat{y}(k)= & \tilde{C}[\theta(k)] \hat{x}_{1}(k), k \in \mathbf{N}
\end{aligned}
$$

where

$$
E_{r[\theta(k)]}=\left[\begin{array}{cc}
0 & 0 \\
0 & I_{r[\theta(k)]}
\end{array}\right] \in \mathbf{R}^{n[\theta(k)] \times n[\theta(k)]} .
$$

\section{BALANCED TRUNCATION}

\section{A. Dissipation inequalities}

The point of departure is a mean square stable MJLS L. The model reduction procedure developed for this class of systems relies on the computation of $U[i]>0$, 
$R[i]>0, i \in \boldsymbol{\Theta}$ such that the following set of dissipation inequalities are satisfied:

$$
\begin{array}{r}
|x|_{U[i]}^{2} \geq \sum_{j \in \boldsymbol{\Theta}} p_{i j} \sum_{l \in \boldsymbol{\Phi}_{\mathbf{i j}}} q_{l}\left(|A[l] x|_{U[j]}^{2}\right)+|C[i] x|^{2}, \\
\forall x \in \mathbf{R}^{n}, \forall i \in \boldsymbol{\Theta}, \\
|x|_{R[i]}^{2}+|f|^{2} \geq \sum_{j \in \boldsymbol{\Theta}} p_{i j} \sum_{l \in \boldsymbol{\Phi}_{\mathbf{i j}}} q_{l}\left(|A[l] x+B[l] f|_{R[j]}^{2}\right), \\
\forall x \in \mathbf{R}^{n}, \forall f \in \mathbf{R}^{m}, \forall i \in \boldsymbol{\Theta}
\end{array}
$$

Inequality (6) will be referred to as the output dissipation inequality and (7) will be referred to as the input dissipation inequality.

1) Output dissipation inequality: Note that (6) are equivalent to the set of LMI's

$U[i] \geq \sum_{j \in \boldsymbol{\Theta}} p_{i j} \sum_{l \in \boldsymbol{\Phi}_{\mathbf{i j}}} q_{l} A[l]^{\prime} U[j] A[l]+C[i]^{\prime} C[i], \quad i \in \boldsymbol{\Theta}$

and imply under zero input the relation

$$
\begin{aligned}
& \mathbf{E}\left[|x(k+1)|_{U[\theta(k+1)]}^{2} \mid x(k), \theta(k)\right]+|y(k)|^{2} \leq \\
& |x(k)|_{U[\theta(k)]}^{2}, \forall x(k) \in \mathbf{R}^{n}, \forall \theta(k) \in \boldsymbol{\Theta} .
\end{aligned}
$$

2) Input dissipation inequality: Relations (7) are equivalent to the set of LMI's

$$
\left[\begin{array}{ll}
W_{11}[i] & W_{12}[i] \\
W_{12}^{\prime}[i] & W_{22}[i]
\end{array}\right] \leq 0, i \in \boldsymbol{\Theta}
$$

where

$$
\begin{aligned}
W_{11}[i] & =-R[i]+\sum_{j \in \boldsymbol{\Theta}} p_{i j} \sum_{l \in \boldsymbol{\Phi}_{\mathbf{i j}}} q_{l} A[l]^{\prime} R[j] A[l] \\
W_{12}[i] & =\sum_{j \in \boldsymbol{\Theta}} p_{i j} \sum_{l \in \boldsymbol{\Phi}_{\mathbf{i j}}} q_{l} A[l]^{\prime} R[j] B[l] \\
W_{22}[i] & =-I+\sum_{j \in \boldsymbol{\Theta}} p_{i j} \sum_{l \in \boldsymbol{\Phi}_{\mathbf{i j}}} q_{l} B[l]^{\prime} R[j] B[l], \quad i \in \boldsymbol{\Theta}
\end{aligned}
$$

and imply

$$
\begin{aligned}
& \mathbf{E}\left[|x(k+1)|_{R[\theta(k+1)]}^{2} \mid x(k), \theta(k)\right] \leq \\
& |x(k)|_{R[\theta(k)]}^{2}+|f(k)|^{2}, \\
& \forall x(k) \in \mathbf{R}^{n}, \forall f(k) \in \mathbf{R}^{m}, \forall \theta(k) \in \mathbf{\Theta} .
\end{aligned}
$$

\section{B. Upper bound on the approximation error}

Theorem 4.1: Consider a mean square stable system $\mathbf{L}$ and matrices $U[i]>0, R[i]>0, i \in \mathbf{\Theta}$ of appropriate dimensions such that the dissipation inequalities (6), (7) are satisfied, and suppose for a particular mode $i^{*} \in \boldsymbol{\Theta}$

$$
U\left[i^{*}\right]=\left[\begin{array}{cc}
\Sigma_{1 i^{*}} & 0 \\
0 & \beta I_{r\left[i^{*}\right]}
\end{array}\right]
$$

and

$$
R\left[i^{*}\right]=\left[\begin{array}{cc}
\Sigma_{2 i^{*}} & 0 \\
0 & \frac{1}{\beta} I_{r\left[i^{*}\right]}
\end{array}\right]
$$

Let $\hat{\mathbf{L}}$ be the reduced order model obtained by truncating (6) the last $r\left(i^{*}\right)$ states corresponding to the mode $i^{*}$ of $\mathbf{L}$. Then, the stochastic $L_{2}$ gain of the error system $\mathbf{E}_{\mathbf{L}, \hat{\mathbf{L}}}$ is bounded from above by

$$
\gamma_{\mathbf{E}_{\mathbf{L}, \hat{\mathbf{L}}}} \leq 2 \beta .
$$

Introduce the matrix

$$
E_{r[i]}=\left[\begin{array}{cc}
0 & 0 \\
0 & I_{r[i]}
\end{array}\right] \in \mathbf{R}^{n[i] \times n[i]} .
$$

and note that $E_{r[i]}=0$ unless $i=i^{*}$. The dynamics of the reduced order system can be written as

$$
\begin{aligned}
\hat{x}(k+1)= & \left(I_{n[\theta(k+1)]}-E_{r[\theta(k+1)]}\right) \\
& (A[\phi(k+1)] \hat{x}(k)+B[\phi(k+1)] f(k)), \\
\hat{y}(k)= & C[\theta(k)] \hat{x}(k), k \in \mathbf{N} .
\end{aligned}
$$

The following variables are introduced to shorten subsequent notation,

$$
\begin{aligned}
z(k) & =x(k)+\hat{x}(k), \\
\delta(k) & =x(k)-\hat{x}(k) \\
h[\phi(k+1)] & =A[\phi(k+1)] \hat{x}(k)+B[\phi(k+1)] f(k), \\
e(k) & =y(k)-\hat{y}(k), k \in \mathbf{N} .
\end{aligned}
$$

One obtains accordingly

$$
\begin{aligned}
z(k+1)= & A[\phi(k+1)] z(k)+2 B[\phi(k+1)] f(k)- \\
& E_{r[\theta(k+1)]} h[\phi(k+1)], \\
\delta(k+1)= & A[\phi(k+1)] \delta(k)+E_{r[\theta(k+1)]} h[\phi(k+1)], \\
e(k)= & C[\theta(k)] \delta(k), k \in \mathbf{N} .
\end{aligned}
$$

According to Lemma 3.1 it is sufficient to find a storage function such that :

$$
\begin{aligned}
& |C[i] \delta|^{2}+\Delta V_{i} \leq 4 \beta^{2}|f|^{2}, \\
& \forall x \in \mathbf{R}^{n[i]}, \quad \forall \hat{x} \in V_{n[i]-r[i]}, \quad \forall f \in \mathbf{R}^{m}, \forall i \in \mathbf{\Theta} \\
& \Delta V_{i}=\sum_{j \in \boldsymbol{\Theta}} p_{i j} \sum_{l \in \boldsymbol{\Phi}_{\mathbf{i j}}} q_{l} V[x(+), \hat{x}(+), j]-V[x, \hat{x}, i] \\
& x(+)=A[l] x+B[l] f \\
& \hat{x}(+)=\left(I_{n[j]}-E_{r[j]}\right)(A[l] \hat{x}+B[l] f) .
\end{aligned}
$$

A quadratic storage function candidate is given by

$V[x, \hat{x}, i]=\beta^{2}|x+\hat{x}|_{R[i]}^{2}+|x-\hat{x}|_{U[i]}^{2}=\beta^{2}|z|_{R[i]}^{2}+|\delta|_{U[i]}^{2}$. 
One needs to verify (10). Let $x \in \mathbf{R}^{n[i]}, \hat{x} \in$ $V_{n[i]-r[i]}, f \in \mathbf{R}^{m}, i \in \boldsymbol{\Theta}$, one has

$$
\begin{aligned}
\Delta V_{i}= & \sum_{j \in \boldsymbol{\Theta}} p_{i j} \sum_{l \in \boldsymbol{\Phi}_{\mathbf{i j}}} q_{l}\left|A[l] \delta+E_{r[j]} h[l]\right|_{U[j]}^{2}+ \\
& \beta^{2} \sum_{i \in \boldsymbol{\Theta}} p_{i j} \sum_{l \in \boldsymbol{\Phi}_{\mathbf{i j}}} q_{l} \mid A[l] z+2 B[l] f+ \\
& -\left.E_{r[j]} h[l]\right|_{R[j]} ^{2}-\beta^{2}|z|_{R[i]}^{2}-|\delta|_{U[i]}^{2} .
\end{aligned}
$$

Expanding the individual terms in the above expressions, one obtains

$$
\begin{aligned}
& \Delta V_{i}=\sum_{j \in \boldsymbol{\Theta}} p_{i j} \sum_{l \in \boldsymbol{\Phi}_{\mathbf{i j}}} q_{l}|A[l] \delta|_{U[j]}^{2}-|\delta|_{U[i]}^{2}+\quad \\
& +\beta^{2} \sum_{j \in \boldsymbol{\Theta}} p_{i j} \sum_{l \in \boldsymbol{\Phi}_{\mathbf{i j}}} q_{l}|A[l] z+2 B[l] f|_{R[j]}^{2}-\beta^{2}|z|_{R[i]}^{2} \\
& +2 \beta \sum_{j \in \boldsymbol{\Theta}} p_{i j} \sum_{l \in \boldsymbol{\Phi}_{\mathbf{i j}}} q_{l}\left|E_{r[j]} h(i, j)\right|^{2} \\
& -2 \beta \sum_{j \in \boldsymbol{\Theta}} p_{i j} \sum_{l \in \boldsymbol{\Phi}_{\mathbf{i j}}} q_{l}\left(E_{r[j]} h(i, j)\right)^{\prime} \\
& (A[l] z+2 B[l] f-A[l] \delta) .
\end{aligned}
$$

Applying the dissipation inequality (6) to the first two terms of (11) gives

$$
\sum_{j \in \boldsymbol{\Theta}} p_{i j} \sum_{l \in \boldsymbol{\Phi}_{\mathbf{i j}}} q_{l}|A[l] \delta|_{U[j]}^{2}-|\delta|_{U[i]}^{2} \leq-|C[i] \delta|^{2} .
$$

Applying the dissipation inequality (7) to the second line in (11) gives

$\beta^{2} \sum_{j \in \boldsymbol{\Theta}} p_{i j} \sum_{l \in \boldsymbol{\Phi}_{\mathbf{i j}}} q_{l}|A[l] z+2 B[l] f|_{R[j]}^{2}-\beta^{2}|z|_{R[i]}^{2} \leq 4 \beta^{2}|f|^{2}$.

For the last term of (11) note that $A[l] z+2 B[l] f-$ $A[l] \delta=2 h[l]$, and that $E_{r[j]}^{2}=E_{r[j]}$. Using the above relations we obtain

$\Delta V_{i}+|C[i] \delta|^{2} \leq 4 \beta^{2}|f|^{2}-2 \beta \sum_{j \in \boldsymbol{\Theta}} p_{i j} \sum_{l \in \boldsymbol{\Phi}_{\mathbf{i j}}} q_{l}\left|E_{r[j]} h[l]\right|^{2}$.

Since $2 \beta \sum_{j \in \Theta} p_{i j} \sum_{l \in \boldsymbol{\Phi}_{\mathbf{i j}}} q_{l}\left|E_{r[j]} h[l]\right|^{2} \geq 0$ relation (10) is satisfied, completing the proof.

It is straight to verify that for the reduced system the dissipation inequalities (6), (7) are satisfied by $\hat{U}[i]=$ $U[i], \hat{R}[i]=R[i]$ when $i \in \boldsymbol{\Theta}, i \neq i^{*}$ and $\hat{U}\left[i^{*}\right]=\Sigma_{1 i^{*}}$, $\hat{R}\left[i^{*}\right]=\Sigma_{2 i^{*}}$. Thus by repeating the above argument one can obtain the twice the sum of the tail result when more than one set of states are truncated and when states in different modes are truncated as well.

\section{REFERENCES}

[1] B. Moore, "Principal component analysis in linear systems, controllability, observability, and model reduction," IEEE Trans. Automat. Contr., vol. AC-26, no. 1, pp. 17-32, Feb. 1981.

[2] K. Glover, "All optimal Hankel-norm approximations of linearmultivariable systems and their $L^{\infty}$-error bounds," Internat. $J$. Control, vol. 39, no. 6, pp. 1115-1193, June 1984.

[3] V. M. Adamjan, D. Z. Arov, and M. G. Krein, "Analytic properties of Schmidt pairs for a Hankel operator and the generalized Schur-Takagi problem," Math. USSR-Sb., vol. 15, no. 1, pp. 3173, 1971.

[4] D. Enns, "Model reduction with balanced realizations: An error bound and a frequency weighted generalization," in Proc. IEEE Conf. Decision Control, Las Vegas, USA, Dec. 1984, pp. 127 132.

[5] U. M. Al-Saggaf and G. F. Franklin, "An error bound for a discrete reduced order model of a linear multivariable system," IEEE Trans. Automat. Contr., vol. AC-32, no. 9, pp. 815-819, Sept. 1987.

[6] D. Hinrichsen and A. J. Pritchard, "An improved error estimate for reduced-order models of discrete-time systems," IEEE Trans. Automat. Contr., vol. 35, no. 3, pp. 317-320, Mar. 1990.

[7] A. Antoulas, Approximation of large-scale dynamical systems. Society for Industrial and Applied Mathematics, 2005.

[8] T. Gudmundsson and A. J. Laub, "Approximate solution of large sparse Lyapunov equations," IEEE Trans. Automat. Contr., vol. 39, no. 5, pp. 1110-1114, May 1994.

[9] I. M. Jaimoukha and E. M. Kasenally, "Krylov subspace methods for solving large Lyapunov equations," SIAM J. Numer. Anal., vol. 31, no. 1, pp. 227-251, Feb. 1994.

[10] C. L. Beck, J. Doyle, and K. Glover, "Model reduction of multidimensional and uncertain systems," IEEE Trans. Automat. Contr., vol. 41, no. 10, pp. 1466-1477, Oct. 1996.

[11] G. Wood, P. Goodard, and K. Glover, "Approximation of linear parameter-varying systems," in Proc. IEEE Conf. Decision Control, Dec. 1996, pp. 406 - 411.

[12] S. Lall and C. L. Beck, "Error-bounds for balanced modelreduction of linear time-varying systems," IEEE Trans. Automat. Contr., vol. 48, no. 6, pp. 946-956, June 2003.

[13] H. Sandberg and A. Rantzer, "Balanced truncation of linear timevarying systems," IEEE Trans. Automat. Contr., vol. 49, no. 2, pp. $217-229$, Feb. 2004.

[14] N. Krasovskii and E. Lidskii, "Analytical design of controllers in systems with random attributes I,II,III," Automat. Remote Control, vol. 22, pp. 1021-1025, 1141-1146, 1289-1294, 1961.

[15] D. Sworder, "Feedback control of a class of linear systems with jump parameters," IEEE Trans. Automat. Contr., vol. 14, no. 1, pp. 9-14, Feb. 1969.

[16] W. M. Wonham, "Random differential equations in control theory," in Probabilistic Methods in Applied Mathematics, A. T. Bharucha-Reid, Ed. Academic Press, 1970, vol. 2, pp. 131212.

[17] O. Costa, M. Fragoso, and R. Marques, Discrete-time Markov Jump Linear Systems. Springer, 2005.

[18] J. P. Hespanha, P. Naghshtabrizi, and Y. Xu, "A survey of recent results in networked control systems," Proc. IEEE, vol. 95, no. 1, pp. 138-162, Jan. 2007.

[19] L. Q. Zhang, B. Huang, and J. Lam., "Model reduction of Markovian jump linear systems," Systems and Control Letters, vol. 50, no. 2, pp. 103-118, Oct. 2003.

[20] J. Chizeck, X.Feng, and K. Loparo, "Stability and control of discrete time jump linear systems," Control Theory Adv. Tech., vol. 7, no. 2, pp. 247-270, 1991. 